\title{
The Case of Lightning Development Panophthalmitis in a Patient with Endocrine Ophthalmopathy
}

\author{
MN Belova* \\ Department of Ophthalmology, Russia \\ *Corresponding author: MN Belova, Department of Ophthalmology, Pugacheva 12-38, Ryazan, Russian Federation 390011, Russia
}

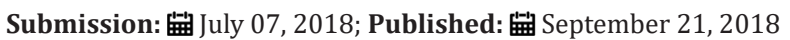

\section{Introduction}

One of the causes of vision and eye loss is purulent inflammation of all the membranes of the eye, the most severe of which is pan ophthalmitis. Pan ophthalmitis is a severe purulent inflammation of the eye, in characterized is acute pain in the eye, complete loss of vision, purulent discharge and exophthalmos. And of the general symptoms are malaise and fever. Anophthalmia can be caused by infection, penetrating wounds of the eye and purulent ulcer of the cornea. Purulent corneal ulcer is acute purulent inflammation of the cornea of the eye with ulceration of infiltrate. The development of purulent corneal ulcer is usually initiated by microtrauma of the corneal epithelium, then infection of the eroded surface occurs, a purulent infiltrate is formed, followed by its disintegration and involvement in the inflammatory process of the anterior parts of the vascular tract of the eye [1].

Purulent corneal ulcer is characterized by the transition of the inflammatory process from the cornea to other eye membranes and the development of complications in the form of pan uveitis and iridocyclitis. In the case of perforation of purulent ulcer, the infection spreads to the inner membranes of the eye which leads to the development of endophthalmitis and pan ophthalmitis [2]. A variety of diseases of the body can contribute to a more rapid flow of the purulent process. Endocrine pathology exacerbates the course of purulent processes in the eye. Endocrine ophthalmopathy is an autoimmune disease manifested by pathological changes in the soft tissues of the orbit with the secondary involvement of the eye [3]. There are three forms of endocrine ophthalmopathy: thyrotoxic exophthalmos, edematous exophthalmos and endocrine myopathy. The most severe of these forms of the disease is edematous exophthalmos, which occurs against the background of hypo-or hyperthyroidism. The beginning of the pathological process is marked by partial intermittent ptosis, followed by rapidly increasing exophthalmos.
With the growth of the pathological process, endocrine ophthalmopathy passes into the stage of decompensation, which is characterized by an aggressive increase in clinical symptoms: exophthalmos reaches extremely high degrees, is accompanied by a non-closing of the eye slit due to sharp edema of periorbital tissues and eyelids, the eye becomes motionless. Because of compression of the ciliary nerves, severe keratopathy or corneal ulcer develops.

\section{Case Presentation}

Patient, 65 years old, female, admitted in the ophthalmology Department on May 27, 2018. On admission, she complained of lack of vision and pain in the left eye, which appeared on May 22. The patient was operated on for nodular toxic goiter in January 2017. In May, the first symptoms of edematous exophthalmos appeared, which reached high degrees (eyeballs mobility with limited and left eye slit did not come to closure). On the night of May 22, she felt a decrease in vision and pain in the eye. But the patient turned to the ophthalmologist only 5 days later, on May 27 and was urgently hospitalized. Diagnosis: corneal ulcer, pan ophthalmitis left eye. Objectively: at admission to the hospital, the visas of the left eye were zero $(0)$, pronounced exophthalmos, chemosis, hyperemia of the eyeball, the cornea is purulent melted, the deeper lying tissues are not visible. Visas of the right eye was 0.3 pronounced exophthalmos, the movements of the eyeball in full range, the cornea is transparent, the deeper lying tissues are not changed [4]. To stop the purulent focus and prevent generalization of the infectious process on the day of admission, evisceration of the left eyeball was carried out. In the postoperative period antiinflammatory therapy was prescribed $[5,6]$.

\section{Conclusion}

This history of the disease demonstrates a casuistic case of rapid, almost lightning-fast development of purulent corneal 
ulcer and pan ophthalmitis against the background of edematous exophthalmos, which ended in the loss of the eye..

\section{References}

1. Brovkina AF (2008) Endocrine ophthalmopathy. Moscow, Russia.

2. Takhchidi GKhP, Yartseva N, Gavrilova NA, Martynov AI, Mkrtumyan AM, et al. (2009) Ophthalmopathology in general diseases, Moscow, Russia.

3. Dedov I, Melnichenko GA (2012) Grave's disease and endocrine ophthalmopathy, Moscow, Russia.
4. Sviridenko NYu (2004) Treatment of Hyperthyroidism in Patients with Endocrine Ophthalmopathy, Moscow, Russia.

5. Prummel MF, Bakker A, Wiersinga WM, Baldeschi L, Mourits MP, et al. (2003) Multi-center study on the characteristics and treatment strategies of patients with Grave's orbitopathy: the first european group on grave's orbitopathy experience. Eur J Endocrinol 148(5): 491-495.

6. Wiersinga W, Bartalena L (2002) Epidemiology and prevention of Grave's ophthalmopathy. Thyroid 12(10): 855-860.
For possible submissions Click Here

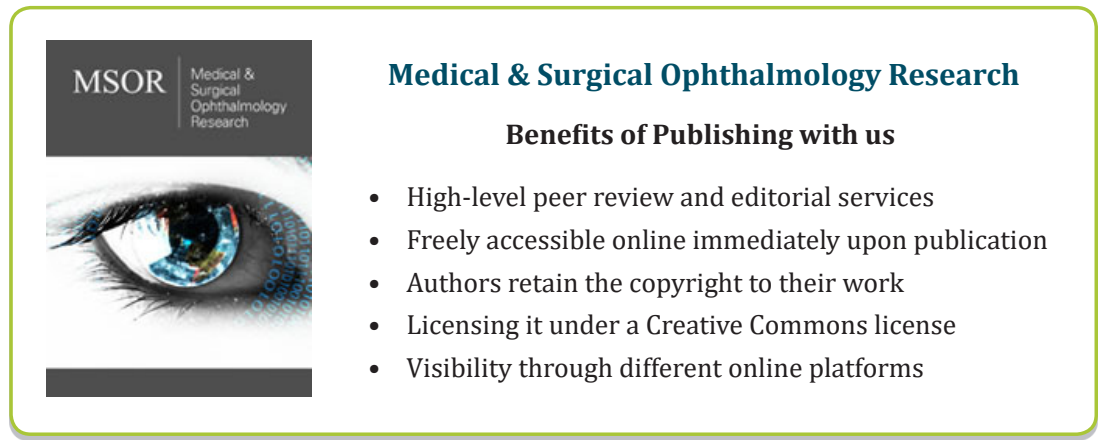

